\title{
Investigation of the Stability Due to Disturbance with SFS Anti-islanding Protection in Multi-DGs System
}

\author{
Hongjun Zhang ${ }^{1, \mathrm{a}}$, Zhenghang $\mathrm{Hao}^{2, \mathrm{~b}^{*}}$ and Zhiqiang $\mathrm{He}^{3, \mathrm{c}}$ \\ ${ }^{1}$ Guizhou University, Guiyang, Guizhou, China \\ ${ }^{2}$ Guizhou University, Guiyang, Guizhou, China \\ ${ }^{3}$ CSR Zhuzhou Electric Locomotive Ltd, Zhuzhou, Hunan, China \\ a718978605@qq.com, b983135026@qq.com, '1262975855@qq.com
}

\begin{abstract}
Keywords: anti-islanding protection, threshold values, system stability, system parameters, multi-DGs system, SFS.

Abstract. This paper presents an improved model of multi-DGs system for analyzing the system stability during grid-connected. The DGs, in the system, are equipped with the Sandia frequency shift (SFS) scheme as anti-islanding protection technique. Relationship of system parameters, such as the length of distribution line, positive feedback with SFS, and stability margins are identified by introducing these variables in the improved model. Through the quantitative analysis, the stability threshold values of the length of distribution line are obtained based on the eigenvalues locus. Although the model is built with SFS proposed as anti-islanding protection technique, the improved model can be adopted to analysis the other anti-islanding protection techniques. This process allows the designers and engineers of renewable energy system to optimize system and ensure stability. Finally, several study cases are carried out through simulation to verity the results obtained from the model. Based on these study cases, general guidelines are recommended to ensure the multi-DGs system stability, which are useful for system analysis, planning and reconfiguration.
\end{abstract}

\section{Introduction}

Multi-DGs system is a system in which a number of Distributed power Generations (DGs) are combined with technological advancements in distribution electronic system. These DGs in system can offer increased reliability and efficient, and help for alleviating energy crisis [1]. Many types of DGs such as fuel-cells, PV cells and micro-turbines are paralleled in the distribution electronic system through power electronic converters [2]. These interface devices make the DGs more flexible in operation and control compared to the conventional electrical machines [3].

Islanding is a special class of faults in multi-DGs system compared with the traditional power grid faults. Sandia frequency shift (SFS) anti-islanding scheme is one of attractive methods for its small non-detection zones (NDZ). In recent publications, the effects of different factors on the SFS protection scheme's efficacy are considered separately. For instance, the impact of DG interface control on anti-islanding protection scheme and NDZ are investigated by Zeineldin and EI-Saadany. This paper extends the works given in [4-7] and presents an improved model of multi-DGs system for DGs with Sandia frequency shift (SFS) anti-islanding protection scheme. Each DG system will have interface controller and LC filter, which is designed to restrain the output harmonics of inverter. The interface controller includes the phase angle shift, current control and voltage control. In order to analysis the effect of parameters, like length of distribution line and DC voltage of DG on the DG's stability, the model introduces the parameters of the length of distribution line $(\mathrm{L})$, DC voltage $\left(\mathrm{V}_{\mathrm{dc}}\right)$ of DG and positive feedback gain (k). Once the model is completed, the stability analysis of multi-DGs system can be finished based on the locus of the eigenvalues, and then the threshold values about length of distribution line and DC voltage of DG can be obtained, respectively. The relation between the system stability and the system parameters can be investigated further through the model. 


\section{Principle of Positive Feedback Anti-Islanding Scheme under Study}

The scheme is designed to add a force to destabilize the islanded DGs when DGs are disconnected from grid. Due to the converters with low-inertia tend to make multi-DGs system sensitive to disturbances [8]. It is critical to study the stability in a multi-DGs system. The previous works about SFS are based on the hypothesis that the destabilizing force is small and has little impact on DG stability when it connected to the grid. However, when many DGs combined in a distribution grid, the interaction will amplify the destabilizing force lead the system to unstable. In order to analysis the interaction quantitatively in multi-DGs system. This paper will study the stability of multi-DGs system with the SFS anti-islanding protection during grid parallel-in by building small signal model of multi-DGs system. The principle of SFS can be expressed as equation (1),

$$
\theta_{f}=\frac{\pi}{2}\left(c f_{0}+k\left(w-w_{0}\right)\right)
$$

where $\theta \mathrm{f}$ is the SFS transformation angles, cf0 is the chopping factor when there is no rotating frequency error and $\omega-\omega 0$ is the difference between the estimated rotating frequency and nominal rotating frequency. Consider a distributed grid in fig. 1 with multiple DGs system. In this figure, the resistance and the inductance of the distribution line1 and line 2 are $R_{L}$ and $X_{L}$, respectively. The multiple DGs system connects to the main grid through CB. Each DG connects to the distribution line through CBs.

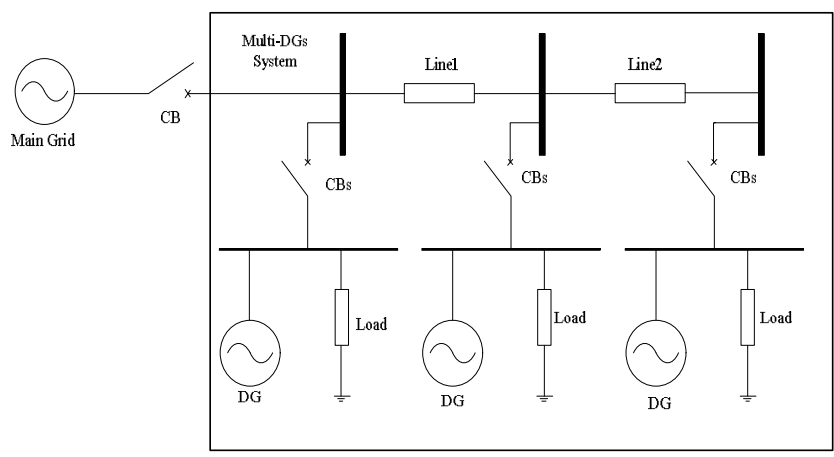

Fig.1 Multi-DGs system under consideration

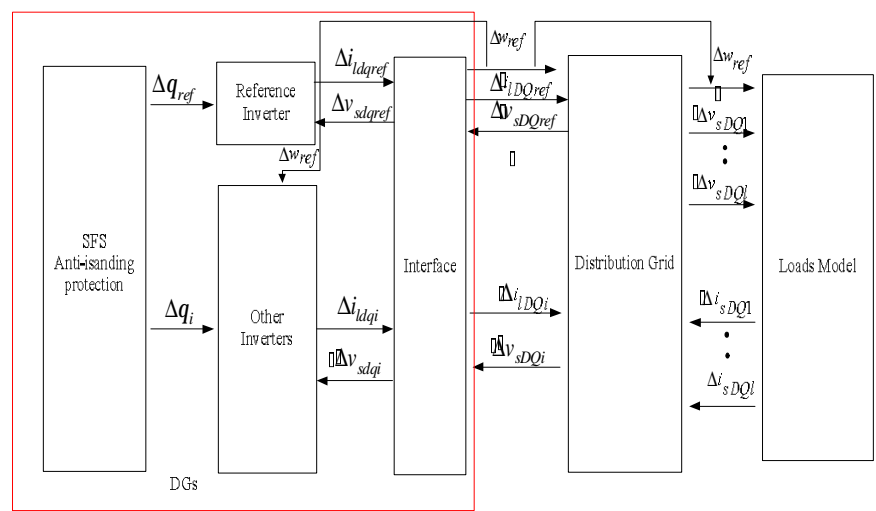

Fig.2 Interconnection block diagram of the Multi-DGs system

The modeling approach can be found in [10], [11] and [12]. However, the existing modeling methods for multiple DGs system are not available to DGs, which is equipped with anti-islanding protection. The whole system is divided into three parts: DGs, distribution grid and loads (Fig. 2). The DG's model includes the interface controller and filter. It's worth to note that each DG is modeled on its individual reference frame whose rotation frequency is set by its local interface controller. In order to keep synchronization, the DGs' model need to be translated to this common reference frame using the transformation technique. The transformation equations are defined in (2) and (3).

$$
\left[\Delta i_{s D Q}\right]=T_{i}\left[\Delta i_{s d q}\right]+T_{c}\left[\Delta \delta_{i}\right]
$$




$$
\left[\Delta v_{s d q}\right]=T_{i}^{-1}\left[\Delta v_{s D Q}\right]+T_{v}\left[\Delta \delta_{i}\right]
$$

where

$$
T_{i}=\left[\begin{array}{cc}
\cos \delta_{0 i} & \sin \delta_{0 i} \\
-\sin \delta_{0 i} & \cos \delta_{0 i}
\end{array}\right], T_{c}=\left[\begin{array}{l}
-\sin \delta_{0 i} I_{s d 0}+\cos \delta_{0 i} I_{s q 0} \\
-\cos \delta_{0 i} I_{s d 0}-\sin \delta_{0 i} I_{s q 0}
\end{array}\right], T_{v}=\left[\begin{array}{c}
-\sin \delta_{0 i} I_{s D 0}-\cos \delta_{0 i} I_{s Q 0} \\
\cos \delta_{0 i} I_{s D 0}-\sin \delta_{0 i} I_{s Q 0}
\end{array}\right] .
$$

The equation (2) and (3) are the positive translation and negative translation. $\delta_{0 \mathrm{i}}$ is the steady-state angle between DGi and common reference frame. $\Delta \delta_{\mathrm{i}}$ is the small incremental of angle between DGi and common reference frame. $\mathrm{I}_{\mathrm{sd} 0}$ and $\mathrm{I}_{\mathrm{sq} 0}$ are the steady-state current is d-q components of DGi, $\mathrm{I}_{\mathrm{sD} 0}$ and $\mathrm{I}_{\mathrm{sQ} 0}$ are the steady-state current is d-q components of reference DG.

\section{Model of Signal Distributed power Generation}

In this paper, the DG1 is selected as common reference frame, whose rotating frequency is $\omega_{\text {ref. }}$ All the other DGs are translated to this common reference frame. Using the transformation technique introduced in (2) and (3), the transferred model of DGi can be expressed as

$$
\begin{aligned}
& Q_{V V}=A \ell R_{V V}+B_{1}\left[\Delta v_{s D Q}\right]+B_{2}[\Delta w] \\
& {\left[\Delta i_{s D Q}\right]=C \mathscr{Q P V}}
\end{aligned}
$$

where

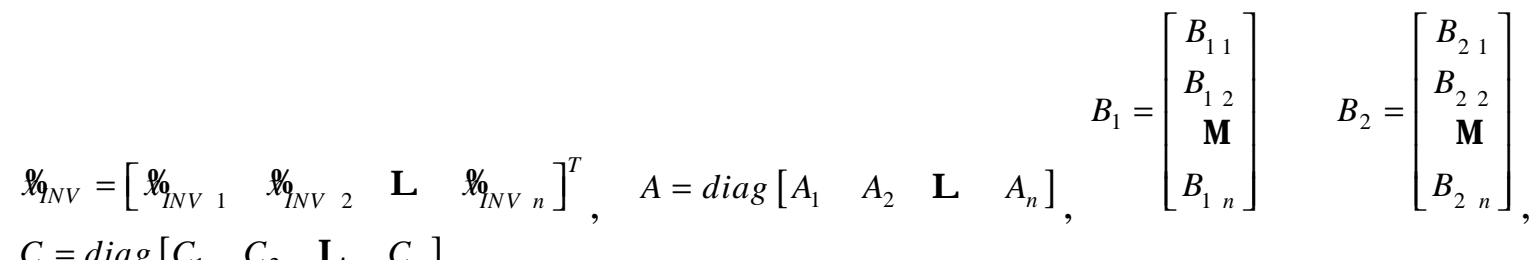

$$
\begin{aligned}
& C=\operatorname{diag}\left[\begin{array}{llll}
C_{1} & C_{2} & \mathrm{~L} & C_{n}
\end{array}\right]
\end{aligned}
$$

\section{Network model}

A distribution grid with $\mathrm{k}$ lines and $\mathrm{l}$ nodes is considered. the model of a distribution grid with $\mathrm{k}$ lines and 1 nodes is given by

$$
\left[\Delta_{\text {line } D Q}^{\mathcal{Q}}\right]=A_{\text {net }}\left[\Delta i_{\text {line } D Q}\right]+B_{\text {net } 1}\left[\Delta v_{s D Q}\right]+B_{\text {net } 2}[\Delta w]
$$

where

$$
\begin{aligned}
& {\left[\begin{array}{lll}
\Delta i_{\text {line } D Q}
\end{array}\right]=\left[\begin{array}{llll}
\Delta i_{\text {line1 } D Q} & \Delta i_{\text {line } 2 D Q} & \cdots & \Delta i_{\text {linek } D Q}
\end{array}\right]^{T},\left[\begin{array}{ll}
\Delta v_{s D Q}
\end{array}\right]=\left[\begin{array}{llll}
\Delta v_{s 1 D Q} & \Delta v_{s 2 D Q} & \cdots & \Delta v_{\text {slDQ }}
\end{array}\right]^{T}}
\end{aligned}
$$

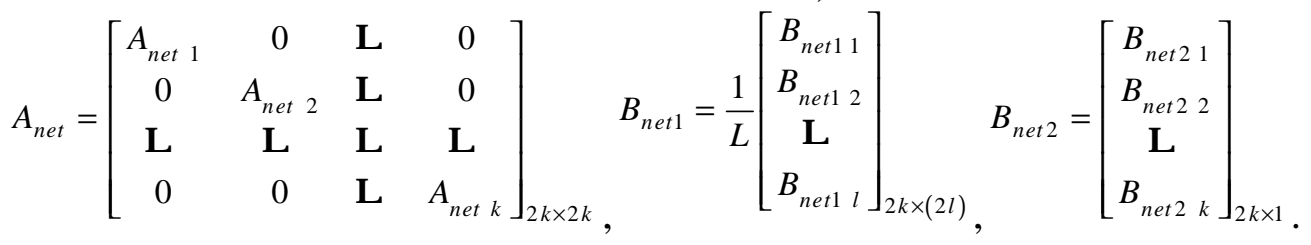

\section{Loads model}

Although, there are many types of load in network, a general $\mathrm{R}_{\mathrm{L}}$ load is considered in this paper. The equations of the $\mathrm{R}_{\mathrm{L}}$ load connected at ith node are

$$
\left[\Delta \mathcal{R}_{\text {load } D Q}^{\mathcal{Q}}\right]=A_{\text {load }}\left[\Delta i_{\text {load } D Q}\right]+B_{\text {load } 1}\left[\Delta v_{\text {s } D Q}\right]+B_{\text {load } 2}[\Delta w]
$$

where 


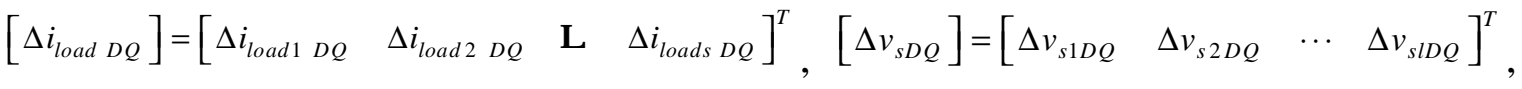

$$
\begin{aligned}
& A_{\text {load }}\left[\begin{array}{cccc}
A_{\text {load } 1} & 0 & \mathrm{~L} & 0 \\
0 & A_{\text {load } 2} & \mathrm{~L} & 0 \\
\mathrm{~L} & \mathrm{~L} & \mathrm{~L} & \mathrm{~L} \\
0 & 0 & \mathrm{~L} & A_{\text {load } s}
\end{array}\right]_{2 s \times 2 s} \quad B_{\text {load } 1}=\left[\begin{array}{c}
B_{\text {load } 11} \\
B_{\text {load } 12} \\
\mathrm{~L} \\
B_{\text {load } 1 s}
\end{array}\right]_{2 s \times(2 l)}, \quad B_{\text {load } 2}=\left[\begin{array}{c}
B_{\text {load } 21} \\
B_{\text {load } 22} \\
\mathrm{~L} \\
B_{\text {load } 2 s}
\end{array}\right]_{2 s \times 1} .
\end{aligned}
$$

\section{Multi-DGs system Model}

Once the model of DG, network and loads is accomplished, the model of multi-DGs system can be obtained by combining each parts models. In order to well define the node voltage, the virtual resistance is introduced between network node and ground. Then the node voltage can be written in terms of currents, it is given by

$$
v_{\text {si } D Q}=r_{N}\left(i_{\text {si } D Q}-i_{\text {loadi } D Q}+i_{\text {linei, }, D Q}\right)
$$

Therefore the node voltage equation of multi-DGs system can be expressed as

$$
\left[\begin{array}{ll}
v_{s D Q} &
\end{array}=R_{N}\left(M_{I N V}\left[\Delta i_{s D Q}\right]+M_{\text {load }}\left[\Delta i_{\text {load } D Q}\right]+M_{\text {net }}\left[\Delta i_{\text {line } D Q}\right]\right)\right.
$$

where, matrix $R_{N}$ is of size $21 \times 21$, whose diagonal elements are equal to $r_{N}$. It is a $21 \times 2 n$ matrix. If the jth DG is connected to the ith node, then the $\mathrm{M}_{\mathrm{INV}}(\mathrm{i}, \mathrm{j})$ is 1 , else is 0 . Similarly, the $\mathrm{M}_{\text {load }}$ is of size $21 \times 2 \mathrm{~s}$ matrix. If jth load is connected to the ith node, then $\mathrm{M}_{\text {load }}(\mathrm{i}, \mathrm{j})$ is -1 , else is 0 . Matrix $\mathrm{M}_{\text {net }}$ of size $2 \mathrm{l} \times 2 \mathrm{k}$ maps the connecting lines onto the network nodes. Here, if ith node is connected with the jth line, and the current flow into the ith node, then the $\mathbf{M}_{\text {net }}(i, j)$ is 1 , else is -1 .

Now, the complete multi-DGs system model (as given in (9)) can be obtained by using the individual subsystem models given by (4), (5), (6), (7) and (9)

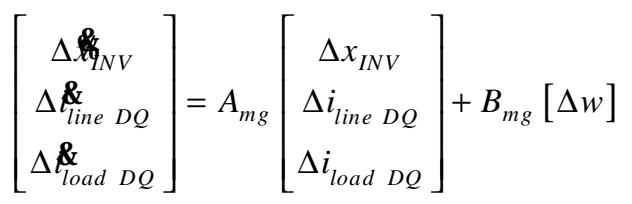

where

$$
B_{m g}=\left[\begin{array}{lll}
B_{2} & B_{\text {net } 2} & B_{\text {load } 2}
\end{array}\right]^{T} \quad, \quad A_{m g}=\left[\begin{array}{ccc}
A_{I N V}+B_{1} R_{N} M_{I N V} C & B_{1} R_{N} M_{\text {net }} & B_{1} R_{N} M_{\text {load }} \\
B_{\text {net } 1} R_{N} M_{\text {INV }} C & A_{\text {net }}+B_{\text {net } 1} R_{N} M_{\text {net }} & B_{\text {net } 1} R_{N} M_{\text {load }} \\
B_{\text {load } 1} R_{N} M_{\text {INV }} C & B_{\text {load } 1} R_{N} M_{\text {net }} & A_{\text {load }}+B_{\text {load } 1} R_{N} M_{\text {load }}
\end{array}\right] .
$$

\section{Simulation Results}

As an example to verify the improved model, a multi-DGs system with 3DGs, 3nodes, 3 loads and 2 transmission lines, is analyzed according the process previously described. The data of the system can be seen in Table. 1 and Table. 2 in Appendix A. The work focuses on analysis the relationship between system parameters and stability.

As DGs are installed along the distribution line, the different lengths of lines have different impact (e.g. the loss of active and reactive power) on system. Fig. 3 and Fig. 4 show the locus of the eigenvalues of distribution line 1 and line2, respectively. It can be seen that the length of lines have significant effect on system stability. 


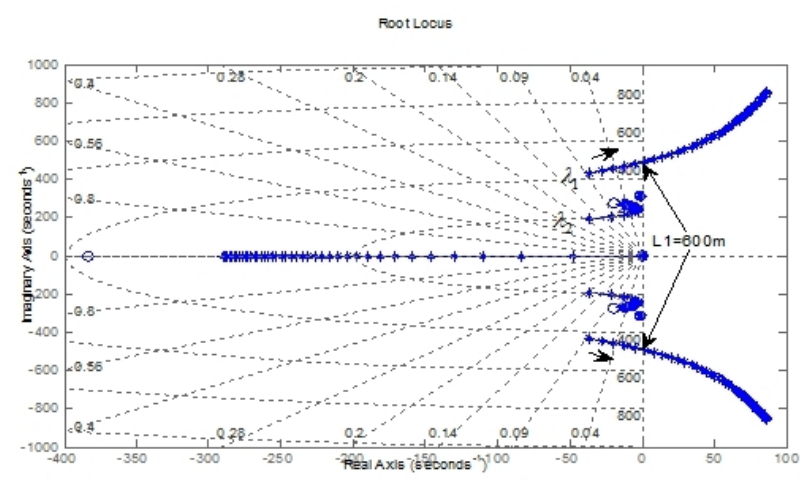

Fig.3 The eigenvalues locus for distribution line1

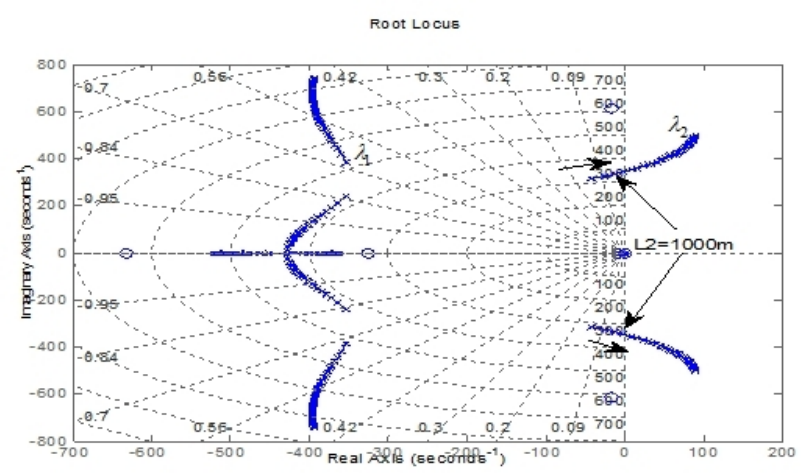

Fig.4 The eigenvalues locus for distribution line2.

Fig. 3 and Fig. 4 illustrate the motion towards the imaginary axis of domain eigenvalues pairs as length of distribution lines are increased. Although, positive feedback gain could adjust the system stability, it also could affect the performance of SFS. Fig. 3 and Fig. 4 also show that high length of distribution line would make system loss stability, with instability occurring at $600 \mathrm{~m}$ and $1000 \mathrm{~m}$ respectively, represented as $\mathrm{L}_{1}{ }^{\max }$ and $\mathrm{L}_{2}{ }^{\max }$. For $\mathrm{L}_{1}<\mathrm{L}_{1}{ }^{\max }, \mathrm{L}_{2}<\mathrm{L}_{2}{ }^{\max }$, the length of distribution line can be annotated as $\mathrm{L}_{1}{ }^{1}$ and $\mathrm{L}_{1}{ }^{1}$, with a corresponding stability margin of 1 . For example, when $\mathrm{L}_{1}=400 \mathrm{~m}$, it can be expressed as $\mathrm{L}_{1}^{400}$.

Case1. Simulation Results validating the analysis of the Distribution line1

In this case, three different length of distribution line1 are given to confirm the analysis result. The length of distribution line1 in states $1 \sim 3$ can be expressed $\mathrm{L}_{1}{ }^{400}, \mathrm{~L}_{1}{ }^{100}$ and $\mathrm{L}_{1}{ }^{-400}$, the length of distribution line 2 is $\mathrm{L}_{2}=200 \mathrm{~m}$ in all states. The negative of stability margin 1 means the system loss stability.

The simulation results are shown in fig. 5(1). In the first state, the terminal frequency responses of DGs are stable. While in the second state and third state, the terminal frequency responses of DG1 are the same with state1, but the terminal frequency responses of DG2 and DG3 became oscillating due to the increasing length of distribution line 1 makes the stability margin 1 reduce from 400 to -400 . It is worth noting that the DG2 and DG3 have already loss stability state3, the stability margin of $\mathrm{L}_{1}$ is -400 . Once the islanding condition occurs at $0.5 \mathrm{~s}$, the SFS can stop the DG in less than 10 cycle at $50 \mathrm{~Hz}$ in all states.

Case2. Simulation Results validating the analysis of the Distribution line2

In this case, the simulation operations are the same with case1. The length of distribution line 2 in states 1 3 can be expressed L2800, L20 and L2-500, the length of distribution line1 is L1=200m in all states. The analysis of the distribution line 2 is validated. 

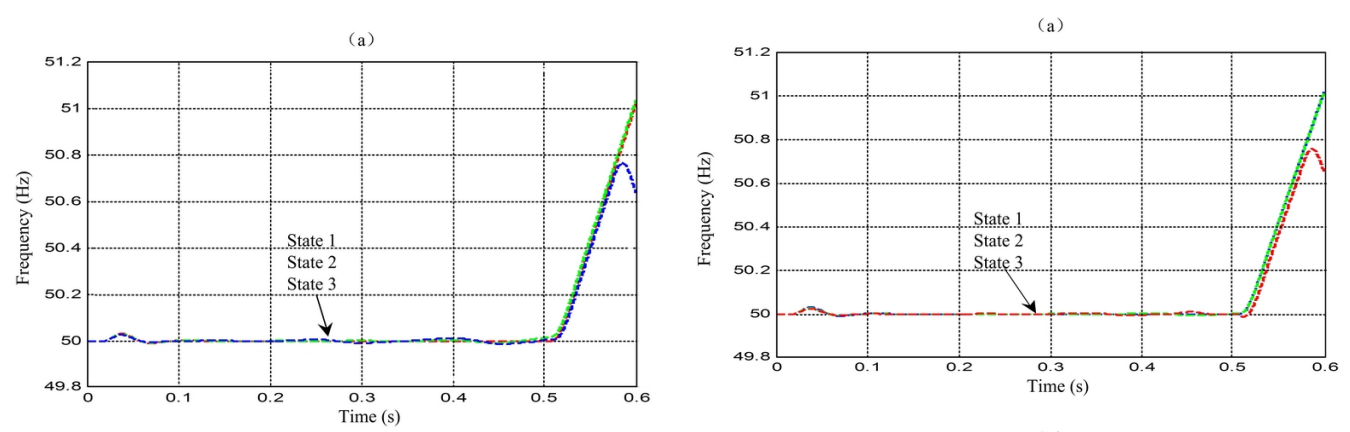

(b)
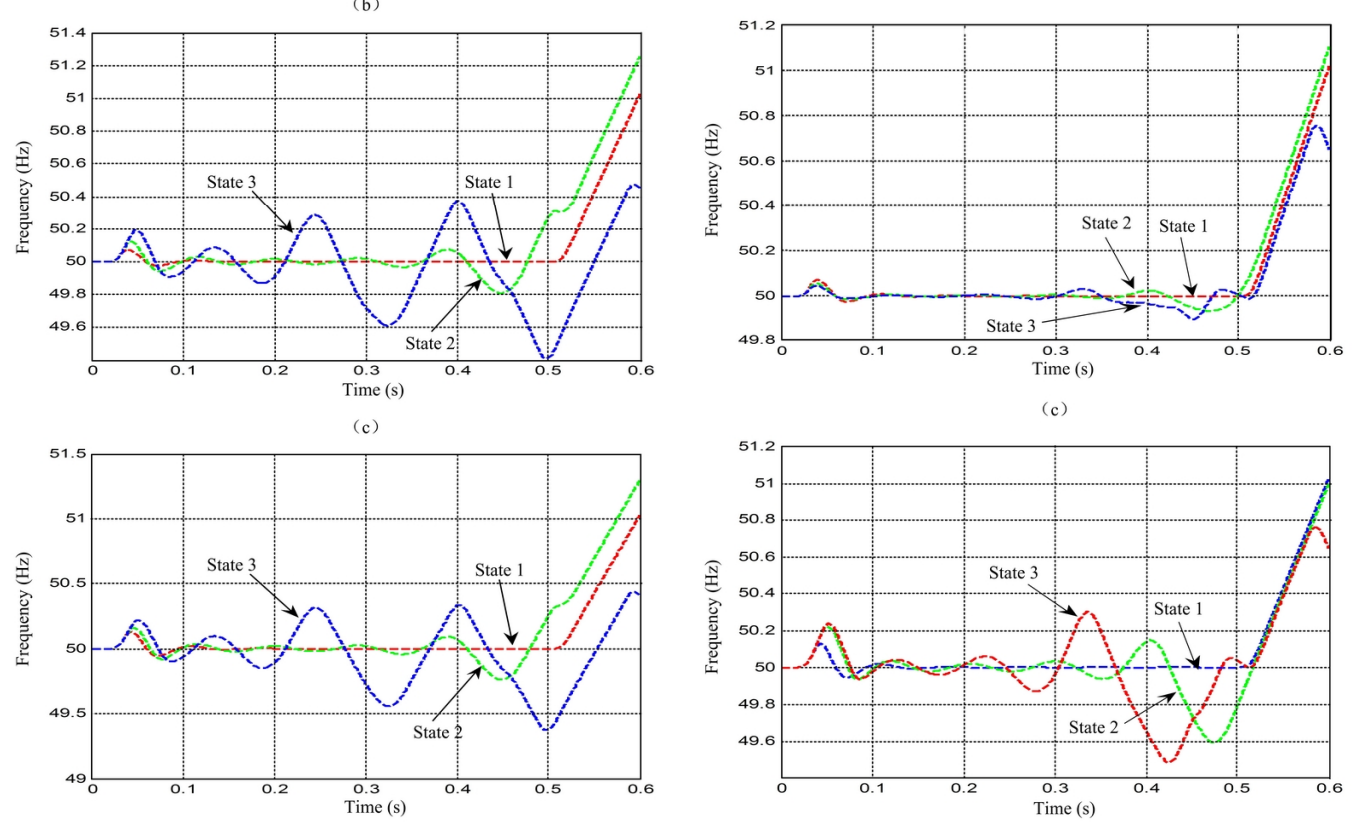

(1)

(2)

Fig.5 (1)The terminal frequency responses of DGs for distribution line1 in case1. (a) Terminal frequency response of DG1. (b) Terminal frequency response of DG2. (c) Terminal frequency response of DG3. The islanding condition is applied at $\mathrm{t}=0.5 \mathrm{~s}$.

Fig.5 (2) The terminal frequency responses of DGs for distribution line2 in case2. (a) Terminal frequency response of DG1. (b) Terminal frequency response of DG2. (c) Terminal frequency response of DG3. The islanding condition is applied at $t=0.5 \mathrm{~s}$.

The simulation results are shown in Fig. 5 (2). The frequency responses of DG1 is the same compared with the Fig. 5 (1-a). It is because the DG1 is connected to the distribution grid directly, changing the length of distribution lines has little influence on frequency responses of DG1. The frequency responses of DG2 has a little oscillation owing to the length of distribution line 2 is increased, but it does not influence the state of system operation.

However, the frequency responses of DG3 are unstable when the distribution line 2 in state 3 , the stability margin are 0 and -500 respectively. Moreover, according the analysis and simulation results, the SFS detection time is affected by increasing distribution line, shown in Fig. 5 and Fig. 6. It means that the system parameters not only affect the system stability, but also effect the SFS detection time. Therefore, the further research is need to model and analysis relationship between length distribution line and SFS detection time.

\section{Conclusions}

The investigations reported in this paper show that the length of distribution lines has a significant influence on stability of multi-DGs system. In order to achieve the quantitative analysis of the influences on multi-DGs system, the model of multi-DGs system is improved based on previous works.

System can be obtained as follow. 
Analysis and simulation on the length of distribution lines show that they can cause instability to the multi-DGs system. In principle, in order to promote the performance of system, the length of distribution line is as short as possible.

Due to the changing on length of distribution lines makes the downstream DGs loss stability, the upstream DGs have little effects. So when adjusting the length of distributed lines, the first choice is to adjust the end of line in the multi-DGs system, because in this way the scope of instability in the system is the smallest.

The suggestions obtained according the model were verified by simulation. It was observed that the simulation results are consistent with the model analysis results. Bedsides, a new problem was found that the system parameters also can affect the SFS detection time. The research about this problem need to be done in the future work.

In conclusion, the results verify that the proposed method can be efficiently implemented for the actual multi-DGs system and able to find out the threshold value of system parameters, which affect the system stability. Due to system stability analysis is important for multi-DGs system design. Consequently, the proposed method is expected to be an effective approach of planning and design for implementation in the renewable energy fields. The suggestions of the paper are useful for the designers and engineers of renewable energy system.

\section{Acknowledgments}

This work is supported by the National Natural Science Foundation of China under Grant No.51467003 and the Science and Technology Project of Guizhou Province under Grant No.[2014]7613.

\section{Appendix A}

Table.1 Distribution line parameters

\begin{tabular}{ccc}
\hline Parameters & Line1 & Line2 \\
\hline Resistance $(\Omega / \mathrm{km})$ & 0.2568 & 0.2568 \\
Inductance $(\mathrm{mH} / \mathrm{km})$ & 2 & 2 \\
Capacitance $(\mathrm{pF} / \mathrm{km})$ & 8.6 & 8.6 \\
\hline
\end{tabular}

Table.2 Parameters for DG

\begin{tabular}{cccc}
\hline Parameters & Value & Parameters & Value \\
\hline $\mathrm{L}_{\mathrm{f}}$ & $1.35(\mathrm{mH})$ & $\mathrm{V}_{\mathrm{dc}}$ & $400(\mathrm{~V})$ \\
$\mathrm{C}_{\mathrm{f}}$ & $50(\mu \mathrm{F})$ & $\mathrm{L}_{\mathrm{c}}$ & $0.35(\mathrm{mH})$ \\
$\mathrm{R}_{\mathrm{f}}$ & $0.1(\Omega)$ & $\mathrm{R}_{\mathrm{c}}$ & $0.03(\Omega)$ \\
$\mathrm{K}_{\mathrm{pi}}$ & 80 & $\mathrm{~K}_{\mathrm{ii}}$ & 200 \\
$\omega_{0}$ & $100(\pi)$ & $\mathrm{K}_{\mathrm{pv}}$ & $2.5 \times 10^{-3}$ \\
$\mathrm{cf}_{0}$ & 0.05 & $\mathrm{k}_{1}$ & 0.5 \\
$\mathrm{k}_{2}$ & 0.05 & $\mathrm{k}_{3}$ & 0.01 \\
\hline
\end{tabular}

\section{References}

[1] Lopes J A, Hatziargyriou N, Mutale J, et al. Integrating distributed generation into electric power systems: A review of drivers, challenges and opportunities[J]. Electric Power Systems Research, 2007, 77(9): 1189-1203. 
[2] Liserre M, Sauter T, Hung J Y. Future energy systems: Integrating renewable energy sources into the smart power grid through industrial electronics [J]. Industrial Electronics Magazine, IEEE, 2010, 4(1): 18-37.

[3] Pepermans G, Driesen J, Haeseldonckx D, et al. Distributed generation: definition, benefits and issues [J]. Energy policy, 2005, 33(6): 787-798.

[4] Zeineldin H H, El-Saadany E F, Salama M M A. Impact of DG interface control on islanding detection and nondetection zones[J]. Power Delivery, IEEE Transactions on, 2006, 21(3): 1515-1523.

[5] Zeineldin H H, Salama M M A. Impact of load frequency dependence on the NDZ and performance of the SFS islanding detection method[J]. Industrial Electronics, IEEE Transactions on, 2011, 58(1): 139-146.

[6] Alaboudy A H K, Zeineldin H H. Islanding detection for inverter-based DG coupled with frequency-dependent static loads[J]. Power Delivery, IEEE Transactions on, 2011, 26(2): 1053-1063.

[7] Wang X, Freitas W, Dinavahi V, et al. Investigation of positive feedback anti-islanding control for multiple inverter-based distributed generators[J]. Power Systems, IEEE Transactions on, 2009, 24(2): 785-795.

[8] Ghaderi A, Kalantar M. Investigation of influential factors on passive islanding detection methods of inverter based distributed generation[C]//Power Electronics, Drive Systems and Technologies Conference (PEDSTC), 2011 2nd. IEEE, 2011: 217-222.

[9] Pogaku N, Prodanovic M, Green T C. Modeling, analysis and testing of autonomous operation of an inverter-based microgrid[J]. Power Electronics, IEEE Transactions on, 2007, 22(2): 613-625.

[10] Wang X, Freitas $\mathrm{W}, \mathrm{Xu} \mathrm{W}$, et al. Impact of DG interface controls on the sandia frequency shift antiislanding method[J]. Energy Conversion, IEEE Transactions on, 2007, 22(3): 792-794.

[11]Energy management in autonomous mocrigrid using stability- constrained droop control of inverters.

[12] WenYuan H, Tao Z, XiaoYu W. Investigation of multi-inverter distributed generation resident Sandia frequency shift anti-islanding method[C]//Advanced Power System Automation and Protection (APAP), 2011 International Conference on. IEEE, 2011, 2: 935-939. 